\title{
Beyond the molecular orbital conception of electronically excited states through the quantum theory of atoms in molecules $\dagger$
}

Cite this: Phys. Chem. Chem. Phys., 2014, 16, 9249

Received 28th January 2014, Accepted 10th March 2014

DOI: $10.1039 / \mathrm{c} 4 \mathrm{cp} 00431 \mathrm{k}$

www.rsc.org/pccp

\author{
David Ferro-Costas, ${ }^{a}$ Ángel Martín Pendás, ${ }^{b}$ Leticia González ${ }^{c}$ and \\ Ricardo A. Mosquera*a
}

\begin{abstract}
We show that the use of the quantum theory of atoms in molecules (QTAIM) in electronically excited states allows expanding the knowledge that the molecular orbital $(\mathrm{MO})$ framework provides about electronic rearrangements. Despite that historical prejudice seemed to preclude the use of QTAIM beyond the electronic ground state, this paper evidences that QTAIM is versatile enough to deal with excited states. As an example, the paradigmatic $n \rightarrow \pi^{*}$ electronic transition of formaldehyde is analyzed. Using QTAIM, an energy partition of excited state energies into atomic and diatomic energies is carried out for the first time. This partition shows that upon electronic excitation the atoms of the $\mathrm{CO}$ bond experience a stabilization in their net energies, accompanied by a destabilization in their interaction, a fact which is in accordance with the idea of populating an antibonding $\pi^{\star} \mathrm{MO}$. The associated $\mathrm{C}-\mathrm{O}$ bond elongation in the $n \pi^{\star}$ state does not involve a change in the $\pi$ atomic populations - as one would expect from a $\pi^{\star}$ orbital - but in the $\sigma$ ones. Moreover, it is also found that the $n \pi^{*}$ state is characterized by a weaker $\mathrm{C}-\mathrm{O}$ interaction energy in comparison to that in the electronic ground state. In order to strengthen this interaction, the electron-electron repulsion between $C$ and $O$ is reduced via a symmetry-breaking of the electron density, causing the $C$ pyramidalization. A topological analysis based on the Laplacian of the electron density and on the electron localization function (ELF) reveals that the $n \rightarrow \pi^{\star}$ transition can be visualized as a rotation of $90^{\circ}$ of the oxygen lone pairs.
\end{abstract}

\section{Introduction}

Understanding the nature of electronically excited states is fundamental to disciplines ranging from basic synthetic organic and inorganic chemistry to nanotechnology and biology. Photoinduced processes are basic to life itself through photosynthesis, and it has become increasingly clear that many biologically important molecules have been subjected to strong natural selection favoring their photostability. ${ }^{1} \mathrm{~A}$ theoretical approach to these problems has traditionally been hindered due to difficulties in solving the time-independent Schrödinger equation for states different from the ground state. However, computational advances in the last decade have started reverting the situation and, with the introduction of multireference methods and

\footnotetext{
${ }^{a}$ Departamento de Química Física, Universidade de Vigo, Facultade de Química, Lagoas-Marcosende s/n, 36310 Vigo, Spain. E-mail: mosquera@uvigo.es

${ }^{b}$ Departamento de Quimica Física y Analítica, Facultad de Quimica, Universidad de Oviedo, 33006-Oviedo, Spain

${ }^{c}$ Institute of Theoretical Chemistry, University of Vienna, Währinger Str. 17, 1090 Vienna, Austria

$\dagger$ Electronic supplementary information (ESI) available. See DOI: 10.1039/ c4cp00431k
}

the developments in linear scaling techniques, ${ }^{2} a b$ initio predictability in excited states for moderately sized systems can be reached. ${ }^{3}$

Conspicuously, despite the increasing wide black-box availability of computational methods to aid practicing chemists, most experiments are designed and/or interpreted in terms of a set of rules that were introduced to understand chemical bonding in electronic ground states. Some of these rules are based on the one-electron approximation inherent to one-determinant descriptions as, for example, the use of molecular orbital (MO) arguments to understand reactivity pathways. Unfortunately, this approximation is sometimes poor, especially in the case of electronic excited states. It is for this reason that a working framework to understand chemical bonding in excited states still needs to be appropriately developed.

Any chemical bonding paradigm for excited states must be able to tackle multireference systems and, as a consequence, it cannot rely on a fixed set of one-electron functions (orbitals). Mainstream ground state chemical bonding theory is still dominated by MO thinking (an intrinsically one-electron scheme). As such, more than often, it offers results strongly dependent on the method used to obtain the approximated wave function because 
MOs are not invariant under orbital transformations. One textbook example is the description of the $\mathrm{C}=\mathrm{C}$ double bond in ethylene. While the Pipek-Mezey localization method ${ }^{4}$ provides a representation based on one $\sigma$ MO and one $\pi$ orbital, the Boys localization method $^{5,6}$ describes the bond by two equivalent banana bond orbitals. Such a situation could be, in a certain way, confusing for some practicing chemists. A possible way out to this ambivalence is to rewrite chemical bonding theory in terms of orbital invariant quantities. These objects can be obtained in terms of reduced density matrices (RDMs), either in position or momentum space. As the chemist's language is developed in the physical space, most of the implementations proposed so far are based on $\mathbf{r}$ dependent RDMs, and these methods are collectively known as real space theories of chemical bonding.

RDMs depend on spin-spatial coordinates and lack atomic information intrinsic to orbitals. Fortunately, Richard F. W. Bader paved the road by showing how atomic, and in general, chemically meaningful regions may be extracted from the $\mathrm{RDMs}^{7,8}$ by examining the topology induced by the electron density, $\rho$. This, so-called Quantum Theory of Atoms in Molecules (QTAIM), has provided deep insights into the nature of the chemical bond for over thirty years, and its methods have been widely generalized to develop what is called Quantum Chemical Topology (QCT). ${ }^{9}$ QTAIM thinking satisfies all of the requirements previously commented: it is invariant under orbital transformations, all of its descriptors are equally defined and obtained for one determinant or correlated wave functions (thus providing a smooth procedure to understand how correlation affects bonding), and it includes an exact energy partitioning into atomic and interatomic contributions. ${ }^{10}$

Traditionally, QTAIM is thought of as a theory of ground state molecules. This historical prejudice roots to the difficulties of the topological procedure to properly define atoms in simple excited states, like the $2 \mathrm{~s} \mathrm{H}$ atom, for which each attraction basin of $\rho$, i.e. each of its local maxima, defines a quantum atom in the theory. A similar situation is found in the ground state of some molecules, e.g. in $\mathrm{Li}_{2}$, which displays a non-nuclear maximum of the electron density at the internuclear midpoint. ${ }^{11}$ However, the controversy on ground state non-nuclear maxima is now over, their existence related to important concepts like F-centers in ionic solids. ${ }^{12}$ Despite that a large class of chemically important molecules do not show excited states with non-nuclear attractors, the use of QTAIM to aid understanding of excited state processes is scarce. ${ }^{13-17}$

To our knowledge, this paper is the first in performing an energetic partition scheme based on QTAIM regions that allows us to understand physically the evolution of the charge density and geometry of a molecule upon excitation. This type of analysis can be extremely helpful to explain, in an orbital invariant language, photophysical and photochemical phenomena. As a practical example, a paradigmatic process in photochemistry, the $\mathrm{n} \rightarrow \pi^{*}$ electronic transition, has been selected. A simple but very well studied molecule, formaldehyde, is chosen to illustrate how QTAIM can explain the molecular changes that take place after the electronic excitation. Three electronic states will be analyzed: the ground state $S_{0}$ and the first singlet $S_{1}$ and triplet $\mathrm{T}_{1}$ states. In the traditional MO language, $\mathrm{S}_{1}$ and $\mathrm{T}_{1}$ are understood as one-electron excitations from a non-bonding $\mathrm{MO}$ centered at the oxygen atom (the $\mathrm{n}$ lone pair) to the $\mathrm{C}-\mathrm{O}$ antibonding $\pi^{*}$ orbital. According to textbook MO machinery, populating the $\pi^{*}$ MO weakens the $\mathrm{C}-\mathrm{O}$ double bond and this manifests as a lengthening of the bond. Concurrently, the hybridization of the $\mathrm{C}$ atom changes from $\mathrm{sp}^{2}$-like to $\mathrm{sp}^{3}$ leading to a $\mathrm{C}$ pyramidalization in any of the two $\mathrm{S}_{1}$ and $\mathrm{T}_{1}$ excited states. ${ }^{18}$ In this work we shall show the ability of the QTAIM to cope with excited states and we will carefully monitor the QTAIM interpretation against the traditional MO explanation, to correlate both views as much as possible.

\section{Methodology}

\subsection{Basic descriptors of the quantum theory of atoms in molecules}

Bader and coworkers showed that the topology induced by the electron density, $\rho(\mathbf{r})$, comes from generalizing the quantum mechanics to subsystems in $\mathbb{R}^{3}$. These subsystems $(\Omega)$ are associated with the atoms in the molecule and they are referred as "atomic basins". In the following, the QTAIM descriptors employed in this paper are briefly revised. Further information can be found in ref. 7, 8 and 19.

Some elements of chemical structure can be identified with critical points of $\rho(\mathbf{r})$. For example, critical points exhibiting three negative curvatures are called nuclear critical points (NCPs), as they are normally placed at, or close to, the position of the nuclei. Critical points with two negative curvatures (and one positive) are called bond critical points (BCPs). The line of locally maximum density linking two NCPs define what is known as the bond path and its presence implies that the corresponding atoms are bonded to one another. The interaction between a given pair of atoms bonded is characterized according to the properties of the electron and energy densities at the BCP.

Two interesting descriptors are defined at the BCP. One is the electron density at that point, $\rho^{\mathrm{BCP}}$, and it is related to the strength of the bonding. The second is the ellipticity, $\varepsilon$, which measures the extent to which density is preferentially accumulated in a given plane containing the bond path, and it is defined as follows

$$
\varepsilon^{\mathrm{BCP}}=\frac{\lambda_{1}}{\lambda_{2}}-1, \quad \text { with } \quad\left|\lambda_{1}\right| \geq\left|\lambda_{2}\right|
$$

where $\lambda_{1}$ and $\lambda_{2}$ are the eigenvalues of the Hessian matrix associated with the eigenvectors which form a plane perpendicular to the bond path. If $\lambda_{1}=\lambda_{2}$ then $\varepsilon=0$ and the bond is cylindrically symmetrical - a situation that can be found e.g. at the $\mathrm{C}-\mathrm{C}$ bonds of ethane and ethine. As such, $\varepsilon^{\mathrm{BCP}}$ has been related to the $\pi$ character, providing reasonable results for most of single and double bonds between carbons.

The average number of electrons associated with the atomic basin $\Omega, N(\Omega)$, can be obtained by

$$
N(\Omega)=\int_{\Omega} \rho(\mathbf{r}) \mathrm{d} \mathbf{r}
$$


These atomic electron populations are usually transformed into net atomic charges by

$$
q(\Omega)=Z-N(\Omega)
$$

where $Z$ is the nuclear charge of the corresponding atom. Moreover, it can be shown that $N(\Omega)$ can be split into two kinds of terms:

$$
N(\Omega)=\lambda_{\Omega}+\frac{1}{2} \sum_{\Omega^{\prime} \neq \Omega} \delta_{\Omega, \Omega^{\prime}}
$$

The term $\lambda_{\Omega}$ is associated exclusively to $\Omega$ and is called localization index. The terms related to the pair of basins, $\delta_{\Omega, \Omega^{\prime}}$, are called delocalization indices and measure the number of electrons shared between $\Omega$ and $\Omega^{\prime}$.

\subsection{Energy partition}

Within the Born-Oppenheimer approximation, the expected value for the molecular electronic energy, $E$, is given by:

$$
E=\langle\Psi|\hat{H}| \Psi\rangle=T+V_{\text {ne }}+V_{\text {ee }}+V_{\text {nn }}
$$

where $\hat{H}$ is the Hamiltonian of the system, $T$ is the kinetic energy, $V_{\text {ne }}$ is the nucleus-electron attraction, and $V_{\text {ee }}$ and $V_{\mathrm{nn}}$ are, respectively, the electron-electron and the nucleusnucleus repulsions.

All these quantities can be written in terms of the electron density of the system, $\rho(\mathbf{r})$ :

$$
\begin{gathered}
V_{\mathrm{ne}}=-\sum_{\alpha=1}^{M} \int \frac{Z_{\alpha} \rho(\mathbf{r})}{\left|\mathbf{R}_{\alpha}-\mathbf{r}\right|} \mathrm{d} \mathbf{r} \\
V_{\text {coul }}=\frac{1}{2} \int \frac{\rho\left(\mathbf{r}_{1}\right) \rho\left(\mathbf{r}_{2}\right)}{\left|\mathbf{r}_{2}-\mathbf{r}_{1}\right|} \mathrm{d} \mathbf{r}_{1} \mathrm{~d} \mathbf{r}_{2}
\end{gathered}
$$

the spin-free first order RDM, $\gamma\left(\mathbf{r}, \mathbf{r}^{\prime}\right)$ :

$$
T=-\frac{1}{2} \int_{\mathbf{r}^{\prime} \rightarrow \mathbf{r}} \nabla^{2} \gamma\left(\mathbf{r}, \mathbf{r}^{\prime}\right) \mathrm{d} \mathbf{r}
$$

and the exchange-correlation component of the diagonal second order RDM, $\Gamma_{\mathrm{xc}}\left(\mathbf{r}_{1}, \mathbf{r}_{2}\right)$ :

$$
V_{\mathrm{xc}}=\frac{1}{2} \int \frac{\Gamma_{\mathrm{xc}}\left(\mathbf{r}_{1}, \mathbf{r}_{2}\right)}{\left|\mathbf{r}_{2}-\mathbf{r}_{1}\right|} \mathrm{d} \mathbf{r}_{1} \mathrm{~d} \mathbf{r}_{2}
$$

where $\mathbf{r}, \mathbf{r}_{1}$ and $\mathbf{r}_{2}$ represent electron coordinates; $M$ is the number of nuclei; and $Z_{\alpha}$ and $\mathbf{R}_{\alpha}$ are the charge and the position vector of the $\alpha$ nucleus. The total electron-electron repulsion, $V_{\text {ee}}$, is split into a classic Coulomb term ( $V_{\text {coul }}$, eqn (7)) and another term associated with the exchange-correlation of the system ( $V_{\mathrm{xc}}$, eqn (9)):

$$
V_{\mathrm{ee}}=V_{\text {coul }}+V_{\mathrm{xc}}
$$

As QTAIM provides a partition of the three-dimensional space into atomic basins, the electron density is also susceptible of partitioning into atomic contributions:

$$
\rho(\mathbf{r})=\sum_{\Omega} \rho_{\Omega}(\mathbf{r})=\sum_{\Omega} w_{\Omega}(\mathbf{r}) \rho(\mathbf{r})
$$

where $\Omega$ represents an atomic basin and the function $w_{\Omega}$ is defined, for the QTAIM partition, by

$$
w_{\Omega}(\mathbf{r})= \begin{cases}1 & \text { if } \mathbf{r} \in \Omega \\ 0 & \text { elsewhere }\end{cases}
$$

Likewise, the RDMs can be also partitioned as

$$
\begin{aligned}
\gamma\left(\mathbf{r}, \mathbf{r}^{\prime}\right) & =\gamma\left(\mathbf{r}, \mathbf{r}^{\prime}\right) \sum_{\Omega} w_{\Omega}\left(\mathbf{r}^{\prime}\right)=\sum_{\Omega} \gamma_{\Omega}\left(\mathbf{r}, \mathbf{r}^{\prime}\right) \\
\Gamma_{\mathrm{xc}}\left(\mathbf{r}_{1}, \mathbf{r}_{2}\right) & =\sum_{\Omega} \sum_{\Omega^{\prime}} w_{\Omega}\left(\mathbf{r}_{1}\right) w_{\Omega^{\prime}}\left(\mathbf{r}_{2}\right) \Gamma_{\mathrm{xc}}\left(\mathbf{r}_{1}, \mathbf{r}_{2}\right) \\
& =\sum_{\Omega} \sum_{\Omega^{\prime}} \Gamma_{\mathrm{xc}}^{\Omega \Omega^{\prime}}\left(\mathbf{r}_{1}, \mathbf{r}_{2}\right)
\end{aligned}
$$

allowing for a whole division of the molecular energy into atomic and diatomic terms. ${ }^{10}$ For instance, using eqn (13) in (8), we obtain:

$$
T=\sum_{\Omega}-\frac{1}{2} \int_{\mathbf{r}^{\prime} \rightarrow \mathbf{r}} \nabla^{2} \gamma\left(\mathbf{r}, \mathbf{r}^{\prime}\right) w_{\Omega}\left(\mathbf{r}^{\prime}\right) \mathrm{d} \mathbf{r}=\sum_{\Omega} T(\Omega)
$$

Similarly, through eqn (11), $V_{\text {ne }}$ is given by

$$
V_{\mathrm{ne}}=\sum_{\Omega} \sum_{\alpha=1}^{M} \int_{\Omega} \frac{-Z_{\alpha} \rho(\mathbf{r})}{\left|\mathbf{R}_{\alpha}-\mathbf{r}\right|} \mathrm{d} \mathbf{r}=\sum_{\Omega} \sum_{\alpha=1}^{M} V_{\mathrm{ne}}(\alpha, \Omega)
$$

Finally, the electron-electron repulsion results in:

$$
\begin{aligned}
V_{\mathrm{ee}}= & \sum_{\Omega} \underbrace{\left\{V_{\text {coul }}(\Omega, \Omega)+V_{\mathrm{xc}}(\Omega, \Omega)\right\}}_{V_{\mathrm{ee}}(\Omega, \Omega)} \\
& +\frac{1}{2} \sum_{\Omega} \sum_{\Omega^{\prime} \neq \Omega} \underbrace{\left\{V_{\text {coul }}\left(\Omega, \Omega^{\prime}\right)+V_{\mathrm{xc}}\left(\Omega, \Omega^{\prime}\right)\right\}}_{V_{\mathrm{ee}}\left(\Omega, \Omega^{\prime}\right)}
\end{aligned}
$$

where the Coulomb components are:

$$
\begin{aligned}
& V_{\text {coul }}(\Omega, \Omega)=\frac{1}{2} \int_{\Omega} \int_{\Omega} \frac{\rho\left(\mathbf{r}_{1}\right) \rho\left(\mathbf{r}_{2}\right)}{\left|\mathbf{r}_{2}-\mathbf{r}_{1}\right|} \mathrm{d} \mathbf{r}_{1} \mathrm{~d} \mathbf{r}_{2} \\
& V_{\text {coul }}\left(\Omega, \Omega^{\prime}\right)=\int_{\Omega} \int_{\Omega^{\prime}} \frac{\rho\left(\mathbf{r}_{1}\right) \rho\left(\mathbf{r}_{2}\right)}{\left|\mathbf{r}_{2}-\mathbf{r}_{1}\right|} \mathrm{d} \mathbf{r}_{1} \mathrm{~d} \mathbf{r}_{2}
\end{aligned}
$$

and the exchange-correlation:

$$
\begin{aligned}
& V_{\mathrm{xc}}(\Omega, \Omega)=\frac{1}{2} \int_{\Omega} \int_{\Omega} \frac{\Gamma_{\mathrm{xc}}\left(\mathbf{r}_{1}, \mathbf{r}_{2}\right)}{\left|\mathbf{r}_{2}-\mathbf{r}_{1}\right|} \mathrm{d} \mathbf{r}_{1} \mathrm{~d} \mathbf{r}_{2} \\
& V_{\mathrm{xc}}\left(\Omega, \Omega^{\prime}\right)=\int_{\Omega} \int_{\Omega^{\prime}} \frac{\Gamma_{\mathrm{xc}}\left(\mathbf{r}_{1}, \mathbf{r}_{2}\right)}{\left|\mathbf{r}_{2}-\mathbf{r}_{1}\right|} \mathrm{d} \mathbf{r}_{1} \mathrm{~d} \mathbf{r}_{2}
\end{aligned}
$$

Once the partition of all the energetic terms associated with the operators comprising the Hamiltonian of the system is done, different terms can be grouped. The collection of all the terms that takes place exclusively in one atomic basin results in the definition of the net energy for that atom, $E_{\text {net }}$,

$$
E_{\text {net }}(\Omega)=T(\Omega)+V_{\text {ne }}(\omega, \Omega)+V_{\text {ee }}(\Omega, \Omega)
$$

where $\omega$ represents the nucleus that belongs to the $\Omega$ basin. Similarly, the interaction energy $\left(V_{\text {int }}\right)$ between 2 atoms can be defined according to the equation,

$$
V_{\text {int }}\left(\Omega, \Omega^{\prime}\right)=V_{\text {nn }}\left(\omega, \omega^{\prime}\right)+V_{\text {ne }}\left(\omega, \Omega^{\prime}\right)+V_{\text {ne }}\left(\omega^{\prime}, \Omega\right)+V_{\text {ee }}\left(\Omega, \Omega^{\prime}\right)
$$


for $\Omega \neq \Omega^{\prime}$ and $\omega \neq \omega^{\prime}$. The introduction of both kinds of energies (net and interaction) into eqn (5) yields the chief equation of the interacting quantum atoms (IQA) energy partition scheme:

$$
E=\sum_{\Omega} E_{\mathrm{net}}(\Omega)+\frac{1}{2} \sum_{\Omega} \sum_{\Omega^{\prime} \neq \Omega} V_{\mathrm{int}}\left(\Omega, \Omega^{\prime}\right)
$$

According to this equation, a molecular system can be analysed in terms of its constituent elements (atoms or even groups of them) and their interactions. This point of view resembles the traditional conception of chemistry before the introduction of quantum mechanics and it does not invoke orbital definitions.

\subsection{Computational details}

All the geometries have been optimized using the Complete Active Space Self Consistent Field (CASSCF) procedure ${ }^{20}$ with an active space of 12 electrons distributed in 10 MOs. The active space is depicted in Fig. 1 and comprises three $\sigma, \sigma^{*}$ pairs, the $\pi_{\mathrm{CO}}, \pi_{\mathrm{CO}}{ }^{*}$ orbital pair, and two lone pairs of electrons of the oxygen atom. For computational sake, the IQA scheme ${ }^{10}$ has been performed using the wave function corresponding to a more modest $\operatorname{CASSCF}(4,3)$ method (restricted to the most relevant lone pair of $\mathrm{O}$ and the $\pi, \pi^{*}$ pair of MOs - see Fig. 1). The energies of each state are computed state-specific. An analysis of the electronic wavefunctions obtained with the $(12,10)$ and $(4,3)$ active spaces reveals that the main configurations contributing to the state are the same. In all cases, the basis set employed is the cc-pVTZ. ${ }^{21}$ All the geometries and energies were obtained using the MOLCAS 7.8 program. $^{22}$

The Molden2AIM ${ }^{23}$ software has been used for the generation of $w f n$ files, needed to carry out the QTAIM analysis of the electron density. This analysis has been done using the AIMPAC package of programs, ${ }^{24}$ as well as using the PROMOLDEN code, ${ }^{10}$ developed by the Quantum Chemistry group of Oviedo University. Additionally, the MULTIWFN software ${ }^{25}$ was used to obtain the Laplacian of the $\rho$ function $\left(\nabla^{2} \rho\right)$ and the Electron Localization Function (ELF). ${ }^{26}$

\section{Results}

\subsection{Geometries}

In order to study the evolution of the $S_{1}$ and $T_{1}$ excited states step by step, a total of five geometries, associated with critical points in different potential energy surfaces (PES), have been considered. Fig. 2 shows the equilibrium geometry in the electronic ground state $\mathrm{S}_{0}$ (denoted as $\min \mathrm{S}_{0}$ ) and the optimized $\mathrm{S}_{1}\left(\min \mathrm{S}_{1}\right)$ and $\mathrm{T}_{1}\left(\min \mathrm{T}_{1}\right)$ minima. As it can be seen, the optimized $\mathrm{S}_{1}$ and $\mathrm{T}_{1}$ minima show pyramidalized $\mathrm{C}$ atoms and $\mathrm{C}-\mathrm{O}$ bonds elongated with respect to the ground state by $c a$. $0.14 \AA$ A. To separate the effect of elongation and pyramidalization, constrained planar optimizations have also been performed in each electronic excited state (planar $\mathrm{S}_{1}$ and planar $\mathrm{T}_{1}$ ). The latter $C_{2 \mathrm{v}}$ geometries correspond to structures with an imaginary vibration frequency associated with the loss of planarity. The relative energy of each critical point in each PES is collected in Table 1. As expected the energy of $T_{1}$ is smaller than that of $S_{1}$ for each geometry.

\subsection{QTAIM analysis in the excited state}

To understand which processes are taking place upon excitation, it is illustrative to analyse the charge density separately in (a) the vertical electronic excitation, (b) the lengthening of the $\mathrm{C}-\mathrm{O}$ and (c) $\mathrm{C}$ pyramidalization steps.

(a) Vertical transition to the excited state. In Fig. 3 the variation of the electron density function, $\Delta \rho(\mathbf{r})$, associated with the $S_{0} \rightarrow S_{1}$ excitation process is depicted. The electron density is mainly depleted (shown in red) from the surroundings of the oxygen atom. This depletion is basically equivalent to the $\rho(\mathbf{r})$ associated with a non-bonding orbital. This electron density is then gained (green) by both the $\mathrm{C}$ and $\mathrm{O}$ atoms, but there is no electron density between the atoms-a fact that can be associated with the idea of populating a $\pi_{\mathrm{C}-\mathrm{O}}{ }^{*} \mathrm{MO}$. As one can see, the analysis of $\Delta \rho(\mathbf{r})$, which is invariant with regard to $\mathrm{MO}$ rotations, naturally provides the same understanding about the charge distribution upon excitation, as the MO theory.

Fig. 4a shows the reorganization of the atomic electron populations in the vertical excitation. As expected, the most important change takes place in the $\mathrm{C}-\mathrm{O}$ moiety, where the oxygen transfers 0.293 electrons to the carbon basin.

The electron density can be also expanded in terms of the natural MO $\chi_{i}$ :

$$
\rho(\mathbf{r})=\sum_{i} n_{i}\left|\chi_{i}(\mathbf{r})\right|^{2}=\sum_{a \in \sigma} n_{a}\left|\chi_{a}(\mathbf{r})\right|^{2}+\sum_{b \in \pi} n_{b}\left|\chi_{b}(\mathbf{r})\right|^{2}
$$

where $n_{i}$ is the occupation number of the natural MO $\chi_{i}$. As also shown in eqn (25), the natural MOs can be grouped

CASSCF $(12,10)$ $\operatorname{CASSCF}(4,3)$

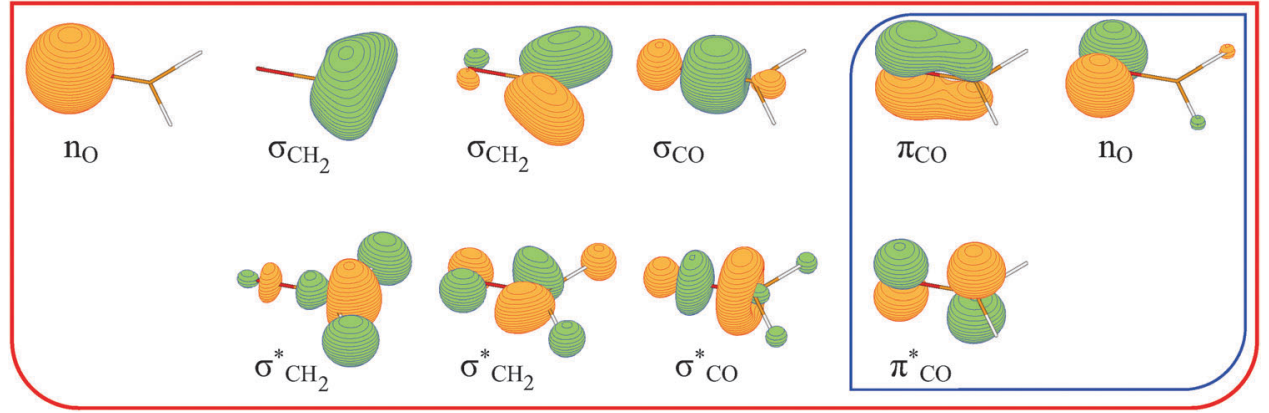

Fig. 1 Natural molecular orbitals of formaldehyde included in the $\operatorname{CASSCF}(12,10)$ active space; here, exemplary for the $S_{0}$ state. The subset selected for the subsequent $\operatorname{CASSCF}(4,3)$ single point calculations is in the blue box. 


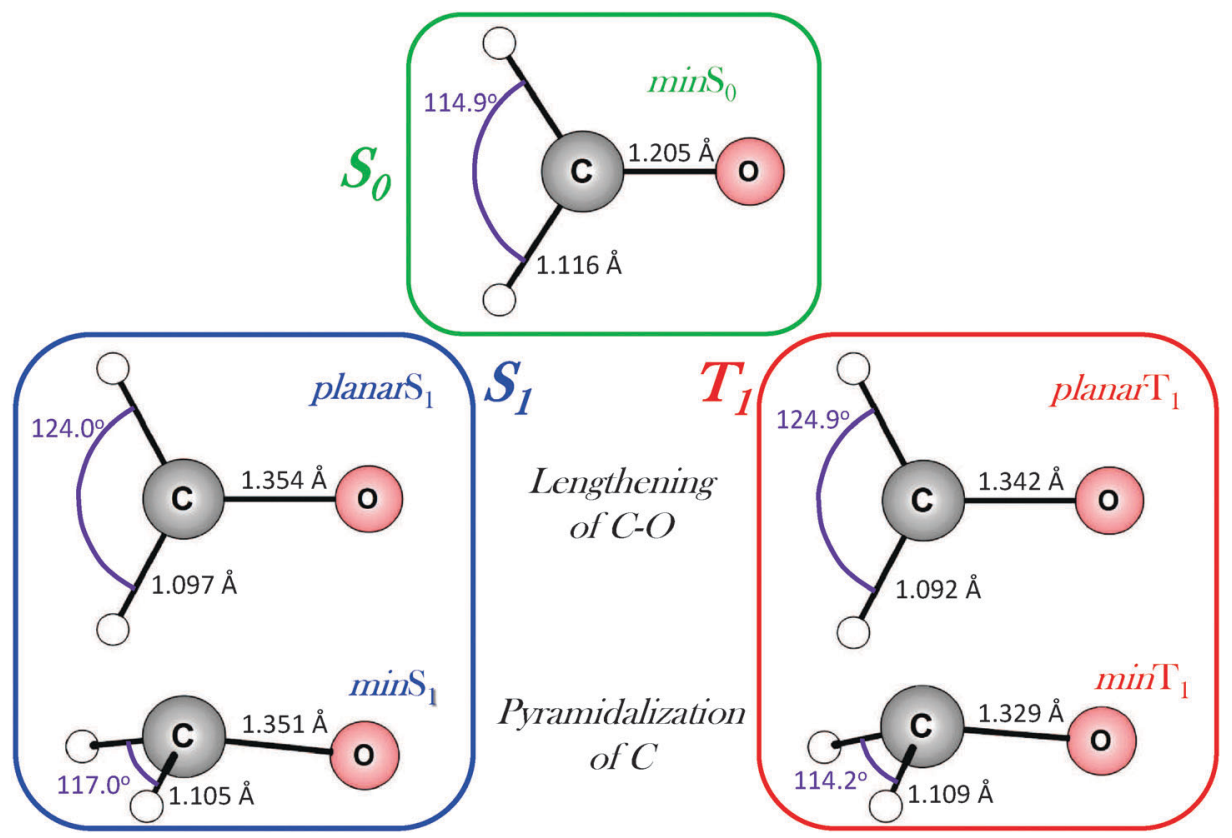

Fig. 2 Equilibrium geometries of formaldehyde in the $S_{0}, S_{1}$ and $T_{1}$ electronic states, as well as planar structures in the $S_{1}$ and $T_{1}$ excited states. Bond distances in $\AA$ and the $\mathrm{H}-\mathrm{C}-\mathrm{H}$ angle in degrees.

Table 1 Relative energies $(\mathrm{eV})$ of formaldehyde obtained at the CASSCF(12,10)/cc-pVTZ level of theory

\begin{tabular}{llll}
\hline & $\mathrm{S}_{0}$ & $\mathrm{~S}_{1}$ & $\mathrm{~T}_{1}$ \\
\hline min $\mathrm{S}_{0}$ & $0.00^{a}$ & 4.52 & 4.26 \\
planar $\mathrm{S}_{1}$ & 0.66 & 4.00 & 3.79 \\
min $\mathrm{S}_{1}$ & 1.07 & 3.94 & 3.67 \\
planar $\mathrm{T}_{1}$ & 0.58 & 4.01 & 3.78 \\
min $\mathrm{T}_{1}$ & 1.12 & 3.96 & 3.66
\end{tabular}

${ }^{a}$ Energy of -114.04552 au.

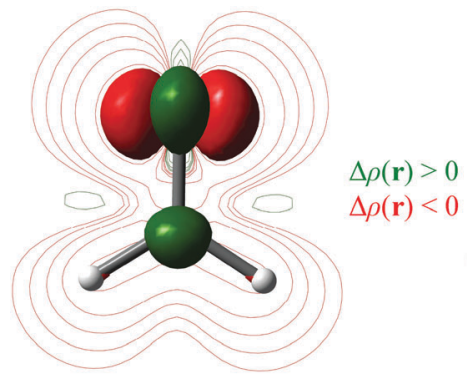

(a) Top view

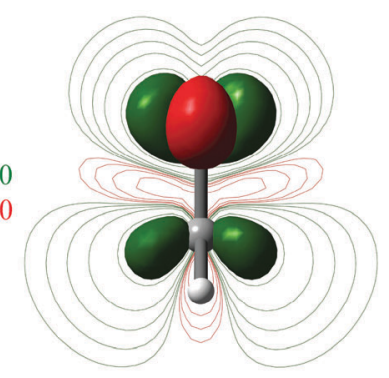

(b) Side view
Fig. 3 (a) Top view and (b) side view of the electron density variation, $\Delta \rho(\mathbf{r})$, in the vertical electronic excitation $\mathrm{S}_{0} \rightarrow \mathrm{S}_{1}$. Isosurfaces of \pm 0.025 au and isolines of $\pm 0.001, \pm 0.002, \pm 0.004, \pm 0.008$, and \pm 0.020 au are shown (positive in green, negative in red).

according to their symmetry ( $\sigma$ or $\pi$ ) and, consequently, the atomic electron population can be split into $\sigma$ and $\pi$ contributions:

$$
N^{\sigma}(\Omega)=\sum_{a \in \sigma} n_{a} \int_{\Omega}\left|\chi_{a}(\mathbf{r})\right|^{2} \mathrm{~d} \mathbf{r}
$$

$$
N^{\pi}(\Omega)=\sum_{b \in \pi} n_{b} \int_{\Omega}\left|\chi_{b}(\mathbf{r})\right|^{2} \mathrm{~d} \mathbf{r}
$$

It should be emphasized that $N^{\sigma}$ and $N^{\pi}$ come from the MO framework and thus they are not physical observables. However, their analysis may still be useful in chemical bonding theory. Moreover, it is interesting to analyze them if we want to find the parallelism between QTAIM results and MO concepts. The $\sigma-\pi$ partitioning of the atomic population in the $S_{0}$ state is shown in Table 2, together with their variations, $\Delta N$, upon vertical excitation. Overall, one " $\sigma$ electron" is converted into one " $\pi$ electron" in the electronic excitation. While o loses 0.759 au from its $\sigma$ distribution, both $\mathrm{C}$ and $\mathrm{O}$ increase their $\pi$ electronic population to 0.484 and 0.466 , respectively (i.e. larger at the $\mathrm{C}$, as expected from the antibonding character of the MO). This means that around $60 \%$ of the oxygen electronic population involved in the electronic transition still belongs to its basin. Similarly, it can be seen that around $40 \%$ of the electronic population gained by the $\mathrm{C}$ comes from the $\mathrm{H}$ atoms, indicating that the transition is not completely described by an $\mathrm{n}_{\mathrm{O}} \rightarrow \pi_{\mathrm{CO}}{ }^{*}$ transition.

Also interesting is to follow the change in the electron density and the ellipticity at the bond critical points ${ }^{7}$ (Table 3 ). The $\mathrm{n}_{\mathrm{O}} \rightarrow \pi^{*}$ electronic transition produces an increase of the electron density in the $\mathrm{C}$ basin, implying a larger electronic repulsion to its environment. As a consequence, the electron density at the BCP of different bonds in which the carbon is involved decreases. Important to note is that although $\rho^{\mathrm{BCP}}$ decreases in the three bonds, only the $\mathrm{C}-\mathrm{O}$ bond distance increases after excitation (see Fig. 2), clearly illustrating that the $\Delta \rho^{\mathrm{BCP}}$ between two electronic states is not enough to predict the geometric reorganization taking place upon excitation. 


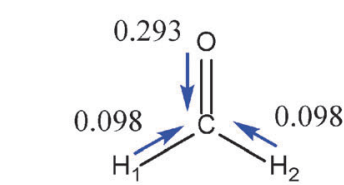

(a)

$\min _{0}$

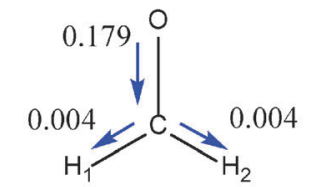

(b)

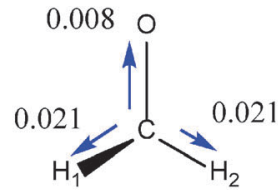

(c)

$\mathrm{S}_{1}$

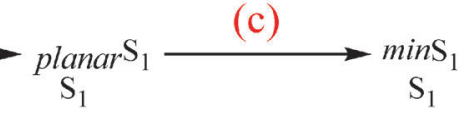

Fig. 4 Reorganization of the electron density (au) from $\min \mathrm{S}_{0}$ (in $\mathrm{S}_{0}$ state) to $\min \mathrm{S}_{1}\left(\right.$ in $\mathrm{S}_{1}$ ) in (a) the vertical electronic excitation $\mathrm{S}_{0} \rightarrow \mathrm{S}_{1}$ in the $m i n \mathrm{~S}_{0}$ geometry, (b) the lengthening of the $\mathrm{C}-\mathrm{O}$ bond in the excited state, and (c) the pyramidalization of the carbonyl carbon.

Table 2 Atomic populations for the $S_{0}$ state (total as well as $\sigma$ and $\pi$ components) and their change after the vertical $\mathrm{S}_{0} \rightarrow \mathrm{S}_{1}$ electronic excitation. All values are in au and referred to the $\min \mathrm{S}_{0}$ geometry

\begin{tabular}{lrrlrrr}
\hline & \multicolumn{1}{c}{$N_{\mathrm{S}_{0}}$} & \multicolumn{1}{c}{$N_{\mathrm{S}_{0}}^{\sigma}$} & \multicolumn{1}{l}{$N_{\mathrm{S}_{0}}^{\pi}$} & \multicolumn{1}{c}{$\Delta N$} & \multicolumn{1}{c}{$\Delta N^{\sigma}$} & $\Delta N^{\pi}$ \\
\hline $\mathrm{C}$ & 4.850 & 4.365 & 0.484 & 0.489 & 0.006 & 0.484 \\
$\mathrm{O}$ & 9.118 & 7.629 & 1.489 & -0.293 & -0.759 & 0.466 \\
$\mathrm{H} 1 / \mathrm{H} 2$ & 1.016 & 1.001 & 0.015 & -0.098 & -0.122 & 0.023 \\
$\Sigma$ & 16.000 & 13.997 & 2.003 & 0.000 & -0.996 & 0.996
\end{tabular}

In passing we note that correlations between $\rho^{\mathrm{BCP}}$ and the bond length should only be done within the same kind of chemical bonds and an electronic excitation does not assure that bonds in different electronic states share the same chemical nature.

The ellipticity increases in both $\mathrm{C}-\mathrm{O}$ and $\mathrm{C}-\mathrm{H}$ bonds, in agreement with the fact that the electron density is accumulated at both sides of the molecular plane over the $\mathrm{C}$ atom, implying that the electron density distribution along the bonds is less cylindrical. This is also a clear example where the association of the ellipticity (or, in general, properties emerging from the electron density) with the bond order (or concepts related to the orbital conception) can be dangerous.

The results of the energy partition following the IQA scheme are collected in Table 4 . The first column refers to the change in energies for the vertical excitation. Accordingly, in the $S_{1}$ excited state the net energies of $\mathrm{C}$ and $\mathrm{O}$ decrease whereas the interaction between them, $\Delta V_{\text {int }}(\mathrm{C}, \mathrm{O})$, becomes very much repulsive $\left(1131 \mathrm{~kJ} \mathrm{~mol}^{-1}\right)$. As $\mathrm{O}$ is negatively charged in the ground state (charge $q_{\mathrm{O}}=-1.118 \mathrm{au}$ ), the withdrawal of electron density from its basins upon excitation stabilizes the atom through the reduction of the electron-electron repulsion in its basin. At the same time, the $\mathrm{C}$ in $\mathrm{S}_{0}$ is positively charged $\left(q_{\mathrm{C}}=+1.150 \mathrm{au}\right)$ and the increment of its atomic electron population increases its nucleus-electron attraction. $\$$ However, this redistribution of the electron density also intensifies the

\footnotetext{
\# The variation experienced by $E_{\text {net }}(C)$ in this vertical electronic excitation may be basically assigned to a charge transfer term. Thus, $\Delta E_{\text {net }}$ roughly corresponds to $-\operatorname{IP}(\mathrm{C}) \cdot \Delta N(\mathrm{C})$, where $\operatorname{IP}(\mathrm{C})$ is the ionization potential of the isolated atom. For example, using the IP experimental value for $\mathrm{C}(11.26 \mathrm{eV})$, we get an estimated $\Delta E_{\text {net }}(\mathrm{C})$ value of $-523 \mathrm{~kJ} \mathrm{~mol}^{-1}$, which satisfactorily compares with our computed value shown in Table $4\left(-606 \mathrm{~kJ} \mathrm{~mol}^{-1}\right)$. This approximation also holds for processes (b) and (c); however, it does not provide good estimations for negatively charged atoms (using electron affinities), where the deformation of the basin plays a more significant role. More details can be found in ref. 27.
}

electron-electron repulsion between both basins (C-O), destabilizing the interaction between them. It can be also of interest to sum up all the inter-atomic interaction energies depending on $\rho(\mathbf{r})$ in what we call a classical potential, $V_{\text {clas }}$, defined as $V_{\text {int }}-V_{\text {xc }}$ (recall eqn (21) and (23)). The variation of $V_{\text {clas }}$ for the CO pair $\left(998.9 \mathrm{~kJ} \mathrm{~mol}^{-1}\right)$ is pretty close to the one obtained by employing a point-charge model (around $877 \mathrm{~kJ} \mathrm{~mol}^{-1}$ ). The same agreement is obtained for the $\mathrm{C}-\mathrm{H}$ bond $\left(35.1 \mathrm{~kJ} \mathrm{~mol}^{-1} v s\right.$. ca. $\left.65 \mathrm{~kJ} \mathrm{~mol}^{-1}\right)$. Moreover, a $\Delta V_{\mathrm{xc}}(\mathrm{C}, \mathrm{O})$ of $132.5 \mathrm{~kJ} \mathrm{~mol}^{-1}$ after vertical excitation (see Table S1 in the ESI $\dagger$ ) indicates that an important part of the $\mathrm{C}=\mathrm{O}$ double bond breaks. In summary, it can be seen that the stabilization of the individual atoms comprised in the bond and destabilization of the interaction, making the bond strength weaker, is in line with the idea of populating an antibonding MO.

Using the CASSCF $(4,3)$ wave function employed in the IQA scheme, localization and delocalization indices ${ }^{7}$ can be obtained (Table 5).§ In the ground state, the most important differences, comparing the expected values of the ideal Lewis structure for the formaldehyde, are found in $\mathrm{C}$ and $\mathrm{O}$ atoms $\left(\lambda_{\mathrm{C}}, \lambda_{\mathrm{O}}\right.$, and $\left.\delta_{\mathrm{C}, \mathrm{O}}\right)$. These differences can be basically justified considering the other resonance form of the $\mathrm{C}=\mathrm{O}$ bond $\left(\mathrm{C}^{+}-\mathrm{O}^{-}\right)$. Upon excitation, the localization indices $(\lambda)$ follow the total basin electron populations ( $c f$. Table 2). Since oxygen suffers a loss of electron density, it becomes less prone to share it, decreasing $\delta_{\mathrm{C}, \mathrm{O}}$. This behavior is again the one expected according to the orbital interpretation because the $\pi^{*}$ MO should entail a reduction in the bond order. Indeed, $\delta_{\mathrm{C}, \mathrm{O}}=1.098$ au for $\mathrm{S}_{1}$ is close to the expected value for a single bond. Therefore, the evolution of $\lambda$ and $\delta$ values could be taken as an indication of the partial heterolytic cleavage of the $\pi$ bond upon vertical electronic excitation. As shown in Table 5, the electrons delocalized in the bond now become localized in one of the two atoms (C).

As it has been previously reported ${ }^{28}$ a change in $\delta_{\Omega, \Omega^{\prime}}$ accompanying vertical excitation may be used, in general, to predict the evolution of the corresponding bond length. Thus, $\delta_{\mathrm{C}, \mathrm{O}}$ decreases $(-0.199 \mathrm{au})$ and $\delta_{\mathrm{C}, \mathrm{H}}$ increases $(0.022 \mathrm{au})$ upon electronic excitation, facts which can be directly related to a stretch and a shrink of the $\mathrm{C}-\mathrm{O}$ and $\mathrm{C}-\mathrm{H}$ bonds, respectively (Fig. 2).

$\S$ The active space used in these calculations introduces correlation basically in the $\mathrm{CO}$ moiety, which is the most important one for our purposes. This means, $\delta_{\mathrm{C}, \mathrm{O}}$ is smaller than the one from Hartree-Fock (HF), while $\delta_{\mathrm{H}, \mathrm{H}}$ and $\delta_{\mathrm{C}, \mathrm{H}}$ are very close to those obtained from HF description. Then, the value of 1.297 au for $\mathrm{CO}$ is clearly a polarized double bond with correlation, whereas 0.908 au is a single $\mathrm{C}-\mathrm{H}$ bond without correlation. 
Table 3 Electron density $\rho(\mathrm{au})$ and ellipticity $\varepsilon$ at the bond critical point (BCP) of $\mathrm{C}-\mathrm{O}$ and $\mathrm{C}-\mathrm{H}$ bonds. The absolute values refer to the $\mathrm{S}_{0}$ state at the min $\mathrm{S}_{0}$ geometry while variations are calculated for (a) the vertical excitation $\mathrm{S}_{0} \rightarrow \mathrm{S}_{1}$, (b) the $\mathrm{C}-\mathrm{O}$ bond elongation, and (c) the $\mathrm{C}$ pyramidalization

\begin{tabular}{|c|c|c|c|c|c|c|c|c|}
\hline & $\rho_{\mathrm{S}_{0}}^{\mathrm{BCP}}$ & $\varepsilon_{\mathrm{S}_{0}}^{\mathrm{BCP}}$ & \multicolumn{2}{|l|}{ (a) } & \multicolumn{2}{|l|}{ (b) } & \multicolumn{2}{|l|}{ (c) } \\
\hline $\mathrm{C}-\mathrm{O}$ & 0.41953 & 0.124 & -0.01196 & 0.015 & -0.10505 & 0.023 & 0.00227 & -0.064 \\
\hline
\end{tabular}

Table 4 Changes in the intra-atomic net energies and the inter-atomic interaction energies ( $\mathrm{KJ} \mathrm{mol}^{-1}$ ) for the processes (a), (b), and (c) considered in Fig. 4. The data correspond to the CASSCF(4,3)/cc-pVTZ level of theory calculation

\begin{tabular}{lrrr}
\hline & (a) & (b) & \multicolumn{1}{c}{ (c) } \\
\hline$\Delta E_{\text {net }}(\mathrm{C})$ & -606.0 & -290.2 & 57.8 \\
$\Delta E_{\text {net }}(\mathrm{O})$ & -203.1 & -398.4 & 10.5 \\
$\Delta E_{\text {net }}(\mathrm{H} 1)^{a}$ & 37.7 & -9.7 & -10.1 \\
$\Delta V_{\text {int }}(\mathrm{C}, \mathrm{O})$ & 1131.4 & 654.5 & -62.2 \\
$\Delta V_{\text {int }}(\mathrm{C}, \mathrm{H} 1)^{a}$ & 36.8 & -32.0 & -5.9 \\
$\Delta V_{\text {int }}(\mathrm{O}, \mathrm{H} 1)^{a}$ & -43.8 & 28.1 & 11.8 \\
$\Delta V_{\text {int }}(\mathrm{H} 1, \mathrm{H} 2)$ & 16.1 & 0.0 & -6.6 \\
${ }^{a}$ Same as H1 for H2. & & & \\
\end{tabular}

(b) Lengthening of the $\mathrm{C}-\mathrm{O}$ bond in the $\mathrm{S}_{\mathbf{1}}$ excited state. After vertical excitation, the nuclei are subjected to new forces and, as a consequence, a geometrical reorganization occurs (in this case the $\mathrm{C}-\mathrm{O}$ bond elongates and the $\mathrm{C}$ pyramidalizes, see $\min \mathrm{S}_{1}$ in Fig. 2). In this section we shall analyse energies and populations obtained within the bond elongation only, i.e. at the planar $\mathrm{S}_{1}$ geometry.

The atomic electron population reorganizations that occur in the $\mathrm{C}-\mathrm{O}$ elongation process are shown in Fig. 4b. The oxygen transfers the electron density to the $\mathrm{C}$ basin (as expected when the $\mathrm{C}-\mathrm{O}$ bond is breaking, since at an infinite $\mathrm{C}-\mathrm{O}$ distance $\mathrm{O}$ should be neutral). A detailed analysis of the $\sigma$ and $\pi$ populations reveals that the nature of the electronic reorganization is practically $\sigma$ (Table 6). Delocalization indices (Table 5) indicate that, in this process, the $\mathrm{C}-\mathrm{O}$ bond order is reduced to a value of 0.995 au. Thus, elongation breaks the double bond, homolytically in the $\pi$ distribution, as no significant changes are observed in $\pi$ populations, and partially heterolytically in the $\sigma$ one.

During the $\mathrm{C}-\mathrm{O}$ bond elongation process, important changes in the values of $\rho^{\mathrm{BCP}}$ and $\varepsilon^{\mathrm{BCP}}$ are not observed, with the exception of the electron density in the $\mathrm{C}-\mathrm{O}$ bond, which obviously decreases as a consequence of the $\mathrm{C}-\mathrm{O}$ bond cleavage (Table 3 ).

In the IQA scheme (Table 4) the same trends found in the excitation process are observed: stabilization of $\mathrm{C}$ and $\mathrm{O}$ net energies and destabilization in their interaction energies. This is again in agreement with the fact that the antibonding character of the $\pi^{*}$ MO is responsible for the elongation. However, our analysis indicates that the lengthening of the $\mathrm{C}-\mathrm{O}$ bond is not accompanied by a transfer of the $\pi$ electron density, but a $\sigma$ one.

(c) Pyramidalization of the $C$ atom in the $S_{1}$ excited state. The electron density reorganization accompanying the pyramidalization (see Fig. 4c) is negligible in comparison with the other

Table 5 Expected values for the localization ( $\lambda$, diagonal values) and the delocalization $(\delta)$ indices for the Lewis standard structure of formaldehyde at $S_{0}$, the calculated values for $\mathrm{S}_{0}$ at $\min \mathrm{S}_{0}$ geometry, and the change of these indices for the $\mathrm{S}_{0} \rightarrow \mathrm{S}_{1}$ excitation, $\mathrm{C}-\mathrm{O}$ bond elongation and $\mathrm{C}$ pyramidalization. All values are in au and calculated at the CASSCF $(4,3) / c c-p V T Z$ level of theory

\begin{tabular}{|c|c|c|c|c|c|}
\hline & $\lambda_{\Omega} \& \delta_{\Omega}, \Omega^{\prime}$ & $\mathrm{C}$ & $\mathrm{O}$ & $\mathrm{H} 1$ & $\mathrm{H} 2$ \\
\hline \multirow[t]{4}{*}{$\mathrm{S}_{0}$ Lewis } & $\mathrm{C}$ & 4.000 & & & \\
\hline & $\mathrm{O}$ & 2.000 & 7.000 & & \\
\hline & H1 & 1.000 & 0.000 & 0.500 & \\
\hline & $\mathrm{H} 2$ & 1.000 & 0.000 & 0.000 & 0.500 \\
\hline \multirow[t]{4}{*}{$\mathrm{S}_{0}$ at $\min \mathrm{S}_{0}$} & $\mathrm{C}$ & 3.305 & & & \\
\hline & $\mathrm{O}$ & 1.297 & 8.389 & & \\
\hline & H1 & 0.908 & 0.112 & 0.458 & \\
\hline & $\mathrm{H} 2$ & 0.908 & 0.112 & 0.051 & 0.458 \\
\hline \multirow[t]{4}{*}{$\mathrm{S}_{0} \rightarrow \mathrm{S}_{1}$ at $\min \mathrm{S}_{0}$} & $\mathrm{C}$ & 0.559 & & & \\
\hline & $\mathrm{O}$ & -0.199 & -0.222 & & \\
\hline & H1 & 0.022 & 0.007 & -0.088 & \\
\hline & $\mathrm{H} 2$ & 0.022 & 0.007 & -0.019 & -0.088 \\
\hline \multirow[t]{4}{*}{$\min \mathrm{S}_{0} \rightarrow$ planar $\mathrm{S}_{1}$ in the $\mathrm{S}_{1}$ state } & $\mathrm{C}$ & 0.147 & & & \\
\hline & $\mathrm{O}$ & -0.103 & -0.033 & & \\
\hline & H1 & 0.029 & -0.036 & 0.001 & \\
\hline & $\mathrm{H} 2$ & 0.029 & -0.036 & 0.000 & 0.001 \\
\hline \multirow[t]{4}{*}{ planar $\mathrm{S}_{1} \rightarrow \min \mathrm{S}_{1}$ in the $\mathrm{S}_{1}$ state } & $\mathrm{C}$ & -0.064 & & & \\
\hline & $\mathrm{O}$ & -0.016 & 0.006 & & \\
\hline & $\mathrm{H} 1$ & -0.006 & 0.010 & 0.032 & \\
\hline & $\mathrm{H} 2$ & -0.006 & 0.010 & 0.002 & 0.032 \\
\hline
\end{tabular}


Table 6 Atomic populations for the $S_{1}$ state (total as well as $\sigma$ and $\pi$ components) at the min $\mathrm{S}_{0}$ geometry and their change in the $\mathrm{C}-\mathrm{O}$ bond elongation. All values are in au

\begin{tabular}{lrrlrrr}
\hline & $N_{\mathrm{S}_{1}}$ & \multicolumn{1}{c}{$N_{\mathrm{S}_{1}}^{\sigma}$} & \multicolumn{1}{c}{$N_{\mathrm{S}_{1}}^{\pi}$} & \multicolumn{1}{c}{$\Delta N$} & \multicolumn{1}{c}{$\Delta N^{\sigma}$} & \multicolumn{1}{c}{$\Delta N^{\pi}$} \\
\hline $\mathrm{C}$ & 5.339 & 4.371 & 0.968 & 0.170 & 0.174 & -0.004 \\
$\mathrm{O}$ & 8.826 & 6.871 & 1.955 & -0.179 & -0.190 & 0.011 \\
$\mathrm{H} 1 / \mathrm{H} 2$ & 0.918 & 0.880 & 0.038 & 0.004 & 0.008 & -0.004 \\
$\Sigma$ & 16.000 & 13.001 & 2.999 & 0.000 & 0.000 & 0.000
\end{tabular}

two processes. Since the total atomic populations remain almost invariable, the localization and delocalization indices (Table 5) as well as the ellipticity and the electron density at the BCPs (Table 3) hardly change. Despite being small, it is interesting to note that the variation of the ellipticity at the BCP in the $\mathrm{C}-\mathrm{O}$ bond (from 0.162 au at planar $\mathrm{S}_{1}$ to 0.097 at $\min \mathrm{S}_{1}$ ) is associated with a larger $\sigma$ character, in agreement with the idea that the $\mathrm{C}$ atom acquires $\mathrm{sp}^{3}$-like hybridisation.

The IQA energy partition (Table 4) for pyramidalization does not show any remarkable stabilization. The most important term arises from the $\mathrm{C}-\mathrm{O}$ interaction, which behaves oppositely to the excitation and $\mathrm{C}-\mathrm{O}$ bond elongation processes. This stabilization, however, is in line with a deformation of the $\mathrm{C}$ electron density to reach a $\mathrm{sp}^{3}$-like hybridisation that occurs to make the bond stronger. In terms of IQA energies this is reflected as a destabilization in $E_{\text {net }}$ and a stabilization in $V_{\text {int }}$.

In order to understand why the $\mathrm{C}$ pyramidalises, it is useful to look at the terms that define $V_{\text {int }}$ (recall eqn (17) and (23)) which are collected in Table S3 of the ESI. $\dagger$ As it can be seen, the origin of the $\mathrm{C}-\mathrm{O}$ stabilization is due to the reduction in the electron-electron repulsion between both atomic basins. Thus, the driving force of the pyramidalization seems to be the reduction of the initial electron-electron repulsion introduced by the vertical excitation (Table S1, ESI $\dagger$ ). This repulsion has its origin (see above) from the $\pi$ contribution of the electron density (see Fig. 3).

For the sake of simplicity, let us consider the ideal spherical out-of-plane $\pi$ electron densities shown in Fig. 5. Taking into consideration only the large interatomic interactions (that is, interactions between spheres in the same side of the plane) depicted as double red arrows, we find that: (i) the $\rho$ with

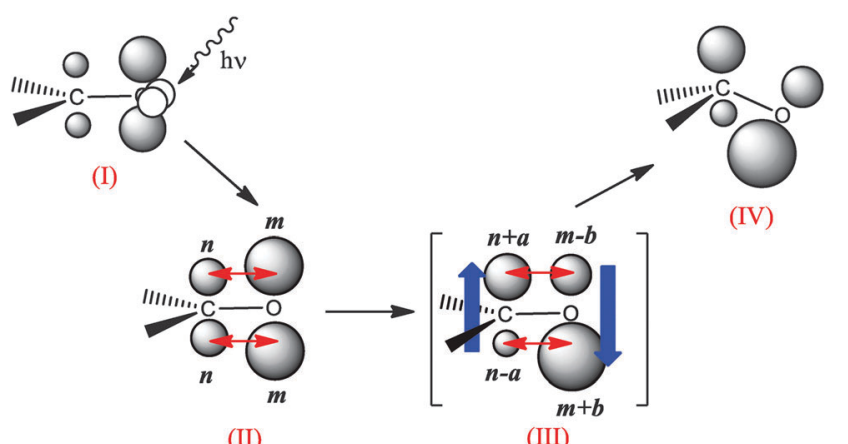

Fig. 5 Scheme of the pyramidalization of the carbonyl carbon. This process takes place in order to reduce the repulsion between the out-ofplane electron densities at $\mathrm{C}$ and $\mathrm{O}$. $\pi$-symmetry in the planar conformation (II) gives rise to $V_{\mathrm{ee}}^{\pi} \propto$ $2 \mathrm{~nm}$ between $\mathrm{C}$ and $\mathrm{O}$ bonds (being $n$ and $m$ the electronic charge of the spheres); (ii) after distortion of the $\pi$ distribution, upon transferring certain $a$ and $b$ amounts of electron density (with $a, b \geq 0$ ) across the plane (as indicated in the imaginary state III), repulsion decreases, since $V_{\mathrm{ee}}^{\pi} \propto 2(n m-a b) ; \uparrow$ and (iii) due to the distortion, the local geometric environment of the atoms is modified, causing the pyramidalization of the atom (IV). In plain English, a symmetry-breaking in the electron density reduces the electron-electron repulsion.

In addition, $V_{\text {clas }}$ and $V_{\mathrm{xc}}$ values for the $\mathrm{C}-\mathrm{O}$ pair $(-71 \mathrm{~kJ}$ $\mathrm{mol}^{-1}$ and $8.8 \mathrm{~kJ} \mathrm{~mol}^{-1}$, respectively) indicate that this process is governed by classical energy terms.

\subsection{Comparison between $S_{1}$ and $T_{1}$}

In the previous section, an analysis of the changes undergone by formaldehyde after excitation from $S_{0}$ to $S_{1}$ has been done. A similar study can be done for the $\mathrm{T}_{1}$ state, at the planar $\mathrm{T}_{1}$ and $\min \mathrm{T}_{1}$ geometries, and the results obtained are very similar (Table S4, ESI $\dagger$ ).

In Fig. 6 , the $\Delta \rho(\mathbf{r})$ function between the $\mathrm{T}_{1}$ and $\mathrm{S}_{1}$ states is shown at planar $\mathrm{S}_{1}$ geometry. The triplet state is characterized by a larger population at the $\mathrm{C}$ basin, which has mainly $\pi$ character (Table 7). This means that the $\pi$ population of $\mathrm{C}$ and O basins are more "alike" in the triplet state than in the singlet (0.964 and 1.967 au vs. 1.006 and 1.920 au, for $\mathrm{C}$ and $\mathrm{O}$ basins, Table 7). According to the mechanism described in Fig. 5, larger repulsions between $\mathrm{C}$ and $\mathrm{O}$ are expected in $\mathrm{T}_{1}$ and the pyramidalization in the triplet state should be slightly larger. Indeed, the HHOC dihedral angle for $\min \mathrm{S}_{1}$ is 22.8 degrees while it is 26.9 degrees for $\min \mathrm{T}_{1}$.

Differences in IQA energetic terms (at planar $\mathrm{S}_{1}$ geometry) are all smaller than $5.0 \mathrm{~kJ} \mathrm{~mol}^{-1}$ in absolute value (Table S5, ESI $\dagger$ ), with the exception of the $E_{\text {net }}(\mathrm{C})$, whose change is $-18.1 \mathrm{~kJ} \mathrm{~mol}^{-1}$. This is due to the increase in the nucleus-electron attraction in the $\mathrm{C}$ basin.

\subsection{Laplacian of the electron density in the excited states}

In QTAIM, the Laplacian of the electron density, $\nabla^{2} \rho(\mathbf{r})$, is typically used to recover the main features of the Lewis and Valence-Shell Electron-Pair Repulsion (VSEPR) models. ${ }^{29}$ Similarly, the electron localization function (ELF) ${ }^{26}$ is known for its ability to analyze the distribution of electron pairs in the molecule. However, the usual formulation of the ELF is only well defined for monodeterminatal wave functions, while $\nabla^{2} \rho(\mathbf{r})$ can be obtained at any level of theory.

Isosurfaces of the Laplacian of the electron density of $S_{0}$ at min $\mathrm{S}_{0}$ and of $\mathrm{S}_{1}$ at $\min \mathrm{S}_{0}$ and $\min \mathrm{S}_{1}$ (Fig. 7) show two interesting facts. First, upon vertical electronic excitation, the $\nabla^{2} \rho<0$ region typically associated with the $\mathrm{O}$ lone pairs rotates 90 degrees around the $\mathrm{C}-\mathrm{O}$ bond. And second, during the pyramidalization, a $\nabla^{2} \rho<0$ region (not associated with any bond) appears in the surroundings of the $\mathrm{C}$ atom. The isosurfaces of $\mathrm{T}_{1}$ at $\min \mathrm{S}_{0}$ and $\min \mathrm{T}_{1}$ are identical to those of $\mathrm{S}_{1}$ and therefore not shown.

T In this situation, $V_{\mathrm{ee}}^{\pi}$ is proportional to $(n+a)(m-b)+(n-a)(m+b)$. 

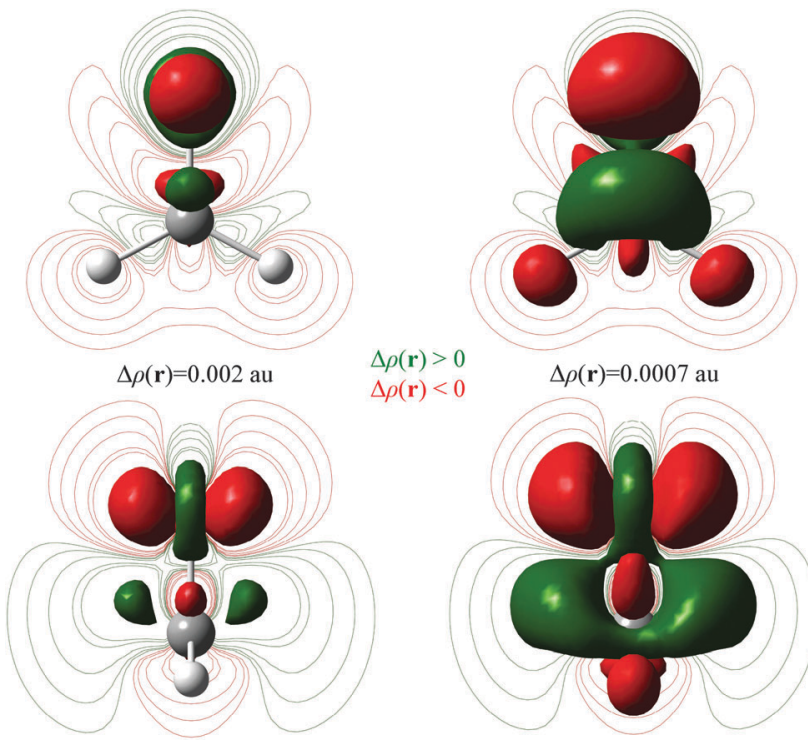

$\Delta \rho(\mathbf{r})=0.0007 \mathrm{au}$

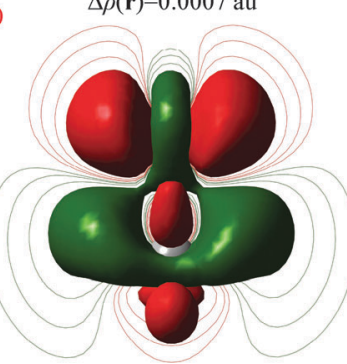

Fig. $6 \rho_{\mathrm{T}_{1}}-\rho_{\mathrm{S}_{1}}$ at the planar $\mathrm{S}_{1}$ geometry. Isosurfaces of \pm 0.002 and \pm 0.0007 au and isolines of $\pm 0.0001, \pm 0.0002, \pm 0.0004, \pm 0.0008$, and \pm 0.001 au are shown (positive in green and negative in red.)

In order to visualize the ELF not only in the $\mathrm{S}_{0}$ state but also in the excited state of formaldehyde, we analyzed the $T_{1}$ state, which can be described with one Slater determinant in an unrestricted Hartree-Fock (UHF) formulation. This is in this case justified because the $S_{1}$ and $T_{1}$ states exhibit similar properties and thus, the ELF analysis may be extended "mutatis mutandis" to describe also the $\mathrm{S}_{1}$ state. Interestingly, the two facts observed with $\nabla^{2} \rho(\mathbf{r})$ are also reproduced by the topology of the ELF, ${ }^{30}$ as observed in Fig. 8.

It is of importance to point that according to the traditional nomenclature of the $S_{0} \rightarrow S_{1}$ transition $\left(n \rightarrow \pi^{*}\right)$, it is expected that the electron density of the lone pairs is transformed into that of a $\pi^{*}$ MO. As it is shown here, the topological analysis of

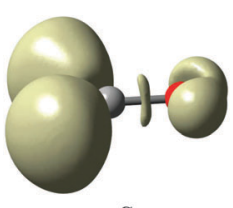

$\underset{\left(\min \mathrm{S}_{0}\right)}{\mathrm{S}_{0}}$

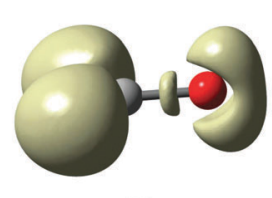

$\mathbf{T}_{1}$

$\left(\min \mathrm{S}_{0}\right)$

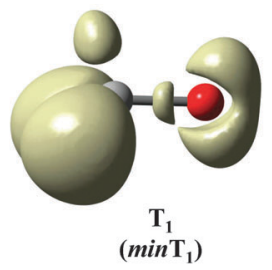

Fig. 80.85 au ELF isosurface for the the $\mathrm{S}_{0}$ (at $\min \mathrm{S}_{0}$ ) and $\mathrm{T}_{1}$ (at $\min \mathrm{S}_{0}$ and $\min \mathrm{T}_{1}$ ) states. The ELF was obtained at the UHF/cc-pVTZ level of theory.

$\rho$ (both QTAIM or ELF) enriches this description, showing that this conversion is caused by a rotation of the $\rho$ associated with the $\mathrm{O}$ lone pairs, what could be interpreted as that the " $\sigma$ " lone pairs become " $\pi$ " lone pairs and this triggers a concomitant weakening of the $\mathrm{C}-\mathrm{O}$ double bond.

The rotation of the $\mathrm{O}$ lone pairs is very relevant, as it appears in a huge number of photophysical processes. It seems reasonable that the $\mathrm{O}$ lone pairs are in a $\sigma$ disposition due to the presence of the $\mathrm{C}-\mathrm{O}$ double bond in the $\mathrm{S}_{0}$ state, even if it maximizes the repulsion between $\mathrm{C}-\mathrm{H}$ bonding pairs and the $\mathrm{O}$ lone pairs. In the $\mathrm{S}_{1}$ electronic arrangement, the repulsion between the $\mathrm{O}$ lone pairs and the $\mathrm{C}-\mathrm{H}$ bonding pairs is minimized.

\section{Conclusions}

The main conclusions may be summarized as follows:

- The QTAIM has proved to be versatile enough to be applied to systems in their electronic excited states, generating results that are in line with firmly-established chemical facts and interpretations. Moreover, QTAIM results have not only been useful as those coming from MO concepts, but even capable of adding details of electronic rearrangements that are not easily deduced from the MO framework.

Table 7 Atomic populations for $S_{1}$ and $T_{1}$ states (total as well as $\sigma$ and $\pi$ components) at the planar $S_{1}$ geometry and their difference $\left(T_{1}\right.$ minus $\left.S_{1}\right)$. All values are in au

\begin{tabular}{lcccccrrrr}
\hline & $N_{\mathrm{S}_{1}}$ & $N_{\mathrm{S}_{1}}^{\sigma}$ & $N_{\mathrm{S}_{1}}^{\pi}$ & \multicolumn{1}{c}{$N_{\mathrm{T}_{1}}$} & $N_{\mathrm{T}_{1}}^{\sigma}$ & $N_{\mathrm{T}_{1}}^{\pi}$ & $\Delta N$ & $\Delta N^{\sigma}$ & \multicolumn{1}{c}{$\Delta N^{\pi}$} \\
\hline $\mathrm{C}$ & 5.509 & 4.545 & 0.964 & 5.556 & 4.551 & 1.006 & 0.048 & 0.005 \\
$\mathrm{O}$ & 8.647 & 6.680 & 1.967 & 8.617 & 6.697 & 1.920 & -0.030 & 0.017 \\
$\mathrm{H} 1 / \mathrm{H} 2$ & 0.922 & 0.888 & 0.035 & 0.913 & 0.877 & 0.037 & -0.009 & -0.011 \\
$\Sigma$ & 16.000 & 13.001 & 2.999 & 16.000 & 13.001 & 2.999 & 0.047 \\
& & & & & &
\end{tabular}
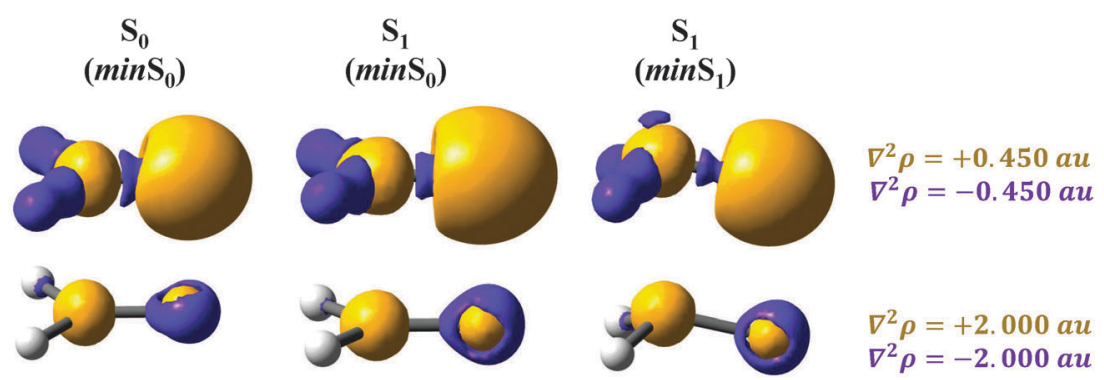

Fig. $7 \nabla^{2} \rho$ isosurfaces ( \pm 0.4 au and \pm 2.0 au; positive in yellow, negative in violet) obtained from the electron density of the $\mathrm{S}_{0}$ (at min $\mathrm{S}_{0}$ ) and $\mathrm{S}_{1}$ (at min $\mathrm{S}_{0}$ and $\min \mathrm{S}_{1}$ ) states. The electron density was obtained at a CASSCF $(12,10) / \mathrm{cc}-\mathrm{pVTZ}$ level of theory. 
- Excitation to the singlet $\mathrm{n} \pi^{*}$ state is accompanied by the stabilization of the individual atoms of the carbonyl bond but with a destabilization of the interaction between them. This fact is in accordance with the idea of populating a $\pi^{*} \mathrm{MO}$, being also an indication that the nature of the $\mathrm{C}-\mathrm{O}$ bond has changed.

- The energy partitions associated with the vertical excitation and the lengthening of the carbonyl bond show the same trend, which could be also ascribed to the antibonding character of the $\pi_{\mathrm{C}-\mathrm{O}}{ }^{*} \mathrm{MO}$. However, the $\mathrm{C}-\mathrm{O}$ bond lengthening does not involve a change in the $\pi$ contribution of the atomic populations, but in the $\sigma$ one.

- The pyramidalization of the $\mathrm{C}$ atom can be understood as a breaking of the symmetry of the electron density in order to reduce the electron-electron repulsion between $\mathrm{C}$ and $\mathrm{O}$ basins.

- The analysis of the electron density (through QTAIM and ELF) reveals that the $n \rightarrow \pi^{*}$ transition may be also understood as a rotation of the oxygen lone pairs to a $\pi$ disposition, accompanied by a synchronous weakening of the $\mathrm{C}-\mathrm{O}$ double bond.

\section{Acknowledgements}

D.F-C. and R.A.M. thank Spanish Ministry of Economy for the project CTQ2010-21500 and A.M.P. for project CTQ2012-31174. D. F-C. also thanks Spanish Ministry of Education for an FPU fellowship and the University of Vigo for the "Estadía en centros de investigación" grant.

\section{References}

1 M. Klessinger and J. Michl, Excited States and Photochemistry of Organic Molecules, VH Publishers, Inc., Toronto, 1995.

2 F. Weigend, M. Kattannek and R. Ahlrichs, J. Chem. Phys., 2009, 130, 164106.

3 L. González, D. Escudero and L. Serrano-Andrés, ChemPhysChem, 2012, 13, 28-51.

4 J. Pipek and P. G. Mezey, J. Chem. Phys., 1989, 90, 4916-4926. 5 J. M. Foster and S. F. Boys, Rev. Mod. Phys., 1960, 32, 300-302.

6 D. A. Kleier, T. A. Halgren, J. H. Hall Jr and W. N. Lipscomb, J. Chem. Phys., 1974, 61, 3905-3919.

7 R. F. W. Bader, Atoms in Molecules: A Quantum Theory, Clarendon Press, Oxford, 1995.

8 R. F. W. Bader, Chem. Rev., 1991, 91, 893-928.

9 P. L. A. Popelier, Struct. Bonding, 2005, 115, 1-56.
10 M. A. Blanco, A. M. Pendás and E. Francisco, J. Chem. Theory Comput., 2005, 1, 1096-1109.

11 J. Cioslowski, J. Phys. Chem., 1990, 94, 5496-5498.

12 P. Mori-Sanchez, J. Recio, B. Silvi, C. Sousa, A. M. Pendas, V. Luaña and F. Illas, Phys. Rev. B: Condens. Matter Mater. Phys., 2002, 66, 075103.

13 R. F. W. Bader, D. Bayles and G. L. Heard, J. Chem. Phys., 2000, 112, 10095.

14 Y.-G. Wang, K. B. Wiberg and N. H. Werstiuk, J. Phys. Chem. A, 2007, 111, 3592.

15 P. B. Coto, D. Roca-Sanjuán, L. Serrano-Andrés, A. M. Pendás, S. Martí and J. Andrés, J. Chem. Theory Comput., 2009, 5, 3032.

16 R. Chávez-Calvillo and J. Hernández-Trujillo, J. Phys. Chem. A, 2011, 115, 13036-13044.

17 V. Tognetti and L. Joubert, Chem. Phys. Lett., 2013, 557, 150-153.

18 N. J. Turro, Modern molecular photochemistry, The Benjamin Cummings, Menlo Park, California, 1978.

19 C. F. Matta and R. J. Boyd, The Quantum Theory of Atoms in Molecules: From Solids State to DNA and Drug Design, Wiley-VCH, 2007.

20 B. O. Roos, P. R. Taylor and P. E. M. Siegbahn, Chem. Phys., 1980, 48, 157-173.

21 T. H. Dunning, J. Chem. Phys., 1989, 90, 1007-1023.

22 F. Aquilante, L. De Vico, N. Ferré, G. Ghigo, P. Malmqvist, P. Neogrády, T. B. Pedersen, M. Pitoñák, M. Reiher, B. O. Roos, L. Serrano-Andrés, M. Urban, V. Veryazov and R. Lindh, J. Comput. Chem., 2010, 31, 224-247.

23 W. Zou, Molden2AIM, http://people.smu.edu/wzou/program/ index.html.

24 R. Biegler-Knig, R. Bader and T.-H. Tang, J. Comput. Chem., 1982, 13, 317.

25 T. Lu and F. Chen, J. Comput. Chem., 2012, 33, 580-592.

26 A. D. Becke and K. E. Edgecombe, J. Chem. Phys., 1990, 92, 5397-5403.

27 A. M. Pendás, M. A. Blanco and E. Francisco, J. Comput. Chem., 2006, 28, 161-184.

28 A. M. Pendás, M. Blanco and E. Francisco, J. Comput. Chem., 2009, 30, 98-109.

29 R. J. Gillespie and P. L. A. Popelier, Chemical Bonding and Molecular Geometry, Oxford University Press, 2001.

30 I. Fourré, B. Silvi, P. Chaquin and A. Sevin, J. Comput. Chem., 1999, 20, 897-910. 